PROFILE

\title{
THE DEVELOPMENT OF A DEGREE QUALIFICATION FOR PARAMEDICS AT CHARLES STURT UNIVERSITY \\ Article No. 990001
}

\section{Bill Lord}

In 1994 the development of a professional degree level qualification for Australian ambulance officers was realised when Charles Sturt University (CSU) and the Ambulance Service of New South Wales (ASNSW) collaborated to establish a Bachelor of Health Science (Pre-Hospital Care). Although pre-hospital qualifications had already made a transition in some states from in-house programs, developed and delivered by the various employing agencies, to the TAFE sector, this course represented the first university based program developed in Australia for pre-hospital practitioners. A similar program at Victoria University of Technology (now Victoria University) was subsequently offered for the first time the following year.

The development and implementation of a degree program was welcomed by many in the industry, although some questioned the relevance of tertiary studies for ambulance officers. This was not surprising given that some individuals are still passionately divided over the decision to transfer nurse education from the hospitals to the higher education setting. The rationale for the shift in education was however, different in each instance. Nursing education in its apprenticeship-based form was an extremely costly exercise for the employing organisations. Hence a major determinant in the move to university-based education involved the assessment of the savings to be gained in shifting the cost of education away from State funded employers to Federally funded institutions of higher education. While this is also a factor influencing decisions about the future of education in the ambulance sector, the rationale for establishing a degree level program for ambulance officers in NSW included a need to progress the professionalism of the discipline and to develop skills that were traditionally lacking in existing vocational training programs. Such skills included knowledge in the area of health services management. As such the course drew heavily on the existing expertise in this area within the Faculty.

Paramedics working at the intensive care level gained credit for $75 \%$ of the course content for prior learning and experience. This left six subjects to complete - five health management subjects and one dealing with clinical issues. Although the health management focus helped to bridge knowledge gaps in this area, both the extent of the management content and the amount of credit allowed for prior learning were later criticised by enrolled and prospective students, as well as sections of the industry.

The first major review of the course occurred in 1998 when CSU decided to offer the Bachelor of Health Science (Pre-Hospital Care) on a full-time basis from the Bathurst campus of the University. The target audience was individuals interested in a career 
in ambulance, hence this program was designed to develop skills, knowledge and attitudes that would enable graduates to seek employment with ambulance services throughout Australia. This initiative required very considerable efforts in developing appropriate course content. It was also essential to ensure that ASNSW could accommodate students during their clinical placements, which aimed to link theory with practice in a workplace setting.

Benchmarks used to develop course content included existing curricula used by ASNSW as well as the recently revised USA EMT-Paramedic: National Standard Curriculum. ${ }^{1}$ Staff from ASNSW as well as the Tasmanian Ambulance Service assisted in the course review process. Most of the resource material developed for the existing distance education program needed to be redesigned to suit the teaching and learning opportunities available in a full-time, on-campus context. A Professional Studies strand of subjects was included, which developed an understanding of the relevance and application of psychological, sociological, legal and ethical issues in prehospital care, together with a greater emphasis on the importance of communication. A new subject that investigated critical thinking, and the diagnostic reasoning and clinical decisions that depend on this process, was also developed.

Teaching and learning strategies were also examined to ensure that the needs of fulltime students without prior industry experience were well supported. Problem focused learning and the development of critical thinking skills were made central to the program. While students are required to recall rules, procedures and protocols in order to implement appropriate patient care, of greater importance is the student's ability to use inductive and deductive processes in order to generate and test hypotheses that may help explain the patient's presentation, and to formulate appropriate and defensible management plans. An understanding of the importance of continuous evaluation and modification of the patient care process is also expected, as is the student's ability to justify their action through the application of appropriate physical and social science concepts.

The use of higher level skills of analysis, evaluation and synthesis to define and explain clinical problems is a valued attribute in higher education, and one which becomes critical in supporting the resolution of problems that are not clearly defined by existing prehospital protocols. Development of these skills is also reliant on the student's ability to locate research articles, and understand the implications of their findings. Such abilities help to develop the life-long learning skills necessary to keep pace with the rapid changes occurring in all human endeavours, particularly the provision of health care to an increasingly demanding public.

A further major course review was completed in 2001. This involved input from the Prehospital Care Course Advisory Committee, comprising representatives of several ambulance services within Australia. This review saw the inclusion of new subjects dealing with mental health, evidence-based practice and advanced pharmacology. The clinical practice component of the course was also strengthened by extending the amount of time available for clinical experience. Students now spend a nominal 480 hours on placement with an ambulance service during the first two years of the course, as well as 160 hours in operating theatres, emergency departments and critical care units of major hospitals in order to develop their advanced life support skills in the final year of the course. Changes to the course in order to produce a 
stronger clinical focus were recognised by a change of nomenclature to Diploma and Bachelor of Clinical Practice (Paramedic).

Student assessment involves a combination of norm-referenced and competencybased assessment, with the later requiring achievement of defined standards of performance. These standards were based on criteria employed by several ambulance services. Policy statements released by the Australian Resuscitation Council also inform the teaching and assessment of clinical practice.

During the clinical placement period students keep a reflective journal as an aid to analysing some of the clinical situations they encounter. When students are placed in a clinical setting in order to integrate their knowledge and skills with actual practice most find the task quite demanding. There are numerous factors that combine to make the provision of patient care somewhat stressful for novice clinicians. Because they have to process so much sensory information novices typically find that they tend to concentrate on the skills being performed, and consequently spend little time analysing other important aspects of the situation. Reflective journals help students to assess their learning by requiring the focus to be on a range of factors influencing the provision of pre-hospital care. This technique also helps to support the student's development as an independent learner.

\section{Student profile}

Most applicants to the full-time Bachelor of Clinical Practice (Paramedic) are school leavers, however a significant number are mature-age students. Some of these applicants have completed nursing or allied health courses at CSU or other universities and have reconsidered career decisions.

Eligible candidates are selected on the basis of defined criteria in combination with Universities Admission Index (UAI) or an equivalent measure. Prospective students must respond to a questionnaire that seeks information about relevant postsecondary training and work experience, and experience with community service organisations. Prospective students must also provide evidence that they understand the nature of prehospital care and must describe the rationale for their choice of study. Each year the applicants include those with first aid experience through work with St John Ambulance, the Rural Fire Service, State Emergency Service or as professional lifeguards. Some have experience as honorary or community ambulance officers. Successful applicants must pass a criminal record check, a requirement imposed by the NSW Department of Health for students undertaking clinical placements in State health institutions.

Selection criteria ensure that potentially capable students are not disadvantaged by a low UAI. However those without relevant prior experience will need a reasonable UAI. In 2002 the UAI cut off was in the low 70s.

\section{The future}

The movement towards a broader education for paramedics is mirrored in other fields, with most other allied health disciplines now adopting graduate entry via an undergraduate program offered by a university. Continuing debate regarding models of education for paramedics will help to inform the future direction of paramedic 
education. However there is already evidence that organisations have moved to establish links with universities to begin the process of developing degree level courses for paramedics.

Rural Ambulance Victoria and Metropolitan Ambulance Service, as well as the ASNSW have developed or are developing employment pathways for graduates from universities offering undergraduate courses in prehospital care. These initiatives appear to have strengthened demand for admission to the full-time course at CSU, with the Bachelor of Clinical Practice (Paramedic) showing very significant increase in demand for places in 2003.

The University is committed to ensuring that graduates meet the expectations of the employing agencies in terms of their knowledge and abilities as novice practitioners. The development of these attributes will be strengthened by a proposal to develop qualified Clinical Mentors through a collaborative agreement between CSU and the ASNSW.

While demand for admission to the full-time course is growing, so too is the size of the part-time cohort. Eligibility for the part-time (distance education) version of the Diploma or Bachelor of Clinical Practice (Paramedic) is restricted to qualified ambulance officers/paramedics who have completed accredited vocational training within a recognised ambulance service to diploma level or equivalent. Full-time medics (Medical Assistants) working with the Australian Defence Forces are also eligible for admission to the Diploma or Bachelor depending on the level of training completed. The part-time cohort now includes practising paramedics from several Australian states and territories, as well as paramedics in the UK and in Canada. The later are enrolled through two affiliate institutions - the Michener Institute and the Southern Alberta Institute of Technology. Medical Assistants who act as paramedics in some areas of Malaysia are also enrolled through Yayasan Pelajaran Mara in Kuala Lumpur.

Very significant demand is also evident for a new combined degree - Bachelor of Nursing/ Bachelor of Clinical Practice (Paramedic). This fully integrate course, which leads to the award of two degrees after four years of full-time study, was approved by the NSW Nurses Registration Board in 2001. The first cohort of students commenced in 2002.

The development of the combined degree was influenced by a proposal in the United Kingdom that recommends a new classification of paramedic - the Practitioner in Emergency Care. ${ }^{2,3}$ This role would depend on a strong nursing and paramedic education, as well as extensive clinical experience in these roles. Similar developments in Canada have reclassified paramedics according to competency profiles, with the most advanced practitioner known as a Critical Care Paramedic. ${ }^{4}$ These proposals call for the development of an extended knowledge and skills base that spans both disciplines in order to prepare the paramedic for new roles in both the hospital and prehospital environment. As such the combined degree offered by CSU reflects these developments by preparing graduates for future challenges and responsibilities in a changing health care environment. 


\section{References}

1. United States Department of Transportation National Highway Traffic Safety Administration. 1998, Emergency Medical Technician-Paramedic (EMT-P): National Standard Curriculum_(on-line)

http://www.nhtsa.dot.gov/people/injury/ems/nsc.htm [Accessed 01.11.02]

2. The Joint Royal Colleges Ambulance Liaison Committee \& Ambulance Service Association. 2000, The future role and education of paramedic ambulance service personnel. JRCALC \& ASA, London.

3. Wood I. Practitioners in emergency care: generic workers or skilled professionals? Pre-hospital Immediate Care. 2000; 4: pp. 132-135.

4. Gibbons MB. 2001, National Occupational Competency Profiles for Paramedic Practitioners. Paramedic Association of Canada, British Columbia.

\section{For further information please contact:}

Bill Lord

Senior Lecturer

School of Public Health

Charles Sturt University

BATHURST NSW 2795

bilord@csu.edu.au 